\title{
Unbalance Rotor Parameters Detection Based on Artificial Neural Network
}

\author{
Mohammad Gohari and Ahmad Kord \\ Faculty of Mechanical Engineering, Arak University of Technology, Iran.
}

(Received 24 August 2016; accepted 31 July 2018)

Unbalance is an important fault that can damage or shut down vital rotary systems such as the gas turbine, compressors, and others, so to avoid this trouble, the balancing process is very crucial, even though it is time-consuming and costly. Thus, having a technique which can predict the unbalance location and its parameters will be valuable and practical. The current study represents a model that can identify the unbalance's mass, radius, and location of the eccentric mass based on the artificial neural network (ANN) model. The inputs of the proposed ANN, which is based on a feed forward with back propagation model, is the bearing acceleration signal in the frequency domain. It has 10 hidden layers with 10 neurons through each layer. The accuracy in prediction was acquired at 96\%, 96\%, and $94 \%$ for the disc number (plane), the eccentric radius, and eccentric mass values, respectively.

\section{INTRODUCTION}

Rotors are important components in rotary systems such as turbines, pumps, compressors, etc. Faults in this component can produce global failure in machinery. Common faults of the rotor dynamic consist of instability because of self-excited vibration, which is oscillation generated by an external load such as a mass unbalance or a bending of the shaft. ${ }^{35}$ Normally, unbalance is known as a fault and occurs in rotational machinery. ${ }^{1}$ Most rotational machines contain unbalance generated during the manufacturing process or installation, and consequently, they need balancing to avoid faults, which will be created after some cycles performed. Commonly given tolerances are used as the thresholds of a balancing operation. The fault of unbalance can be created by various phenomenon. In fact, rotor unbalance is a situation in which the centre of mass of a rotating component, normally the shaft and its fixed components, like disks and blades etc., is not coincident with the centre of rotation. In the operation condition, rotors can never be exactly balanced due to manufacturing errors such as generated porosity in casting, non-homogenous density of material, or the loss of material through forming. ${ }^{17}$

Sometimes, unbalance is created by the operation of rotary machines in long term use. For example, in a gas turbine, unbalance might happen due to blade damages, internal defects during blade manufacturing, inaccurate installation after overhaul of assembly, cavitation, or a corrosive fluid.

Nowadays, rotating machinery should be more reliable and unpredicted fault maintenance is a big challenge for companies. This issue has gotten more important due to consumers asking machinery producers to design and manufacture machines with shorter turnaround times because they would like to decrease cost. ${ }^{2}$ Although reliability levels are increased, unbalance remains as a rotor fault, which needs unscheduled maintenance. ${ }^{3}$

The localization of unbalance and finding eccentric mass ra- dius values accurately in the complex machines provides good information for technicians to access fault points and do the balancing directly. The traditional balancing techniques such as borescoping and multi-plane balancing are time consuming procedures.

Many researches were conducted on unbalance in rotational machines and this issue already remains an open area for the technical researchers. Some of them focused on the relationship between misalignment and unbalance, ${ }^{4,5}$ and some of them report on unbalance diagnosis. ${ }^{6,7}$ In addition, a number of investigators try to employ simulation techniques such as unbalance prediction, ${ }^{8,9}$ and indoor experimental approaches. $^{10,11}$ The details of existing unbalance detection methods are reviewed in several publications. ${ }^{12-15}$ Also, fault diagnosis of a rotor-bearing system for misalignment and unbalance was investigated in a steady state. ${ }^{36}$

In spite of some techniques available to diagnosis unbalance, localization of the unbalance and the identification of how much is the eccentric masses generating unbalances are very useful. One of the attempts that tried to employ mode shapes and modal masses instead of a test run was carried out by Krodkiewski et al. ${ }^{16}$ They established a mathematical model for a multi-bearing rotor system. This idea has been developed for a flexible rotating machine via a single run. ${ }^{17,18}$ Furthermore, the shaft deflection measurement was utilized to reach the position of the unbalance. ${ }^{19,20}$ The frequency response of the system, which was simulated by the NASTRAN software, was applied in a prediction of the unbalance position. ${ }^{21}$

Finite element modelling has been employed to study variation of stiffness, which is generated by faults such as crack, impact, rubbing, and other. These variations can be used for finding the localization of the unbalance. ${ }^{22,23}$

Artificial neural network (ANN) is one of the intelligent techniques that can be used for prediction in the systems in which the mathematical model is not clear or linear. For instance, it was performed for fault detection in the bear- 
ings, ${ }^{24-26,37}$ shaft mode shapes classification for the recognition of cracks, ${ }^{27}$ fault detection and classification in the rotary machines via Neuro-fuzzy tools, ${ }^{28}$ failure identification and detection in the gears and bearings by Self Adaptive Growing Neural Network (SAGNN). ${ }^{26}$ In addition, the number of the systems equipped by a hybrid of ANN and fuzzy systems have been used to detect unbalance in the rotary system, ${ }^{28}$ to classify faults of the turbo generators, ${ }^{28}$ and to approximate the correction masses value for the balancing rotor. ${ }^{29}$ The integration of ANN with Wavelet, Possibility Theory, and Taguchi were tried in rotary system diagnosis. ${ }^{38-40}$ The unbalance localization via ANN was developed for a multi-disc rotor. It could identify the plane of the unbalance. ${ }^{30}$ An algebraic method had been operated to identify the unbalance in the system for its active controlling. ${ }^{31}$ Active systems can cancel the unbalance effects by means of some external forces in the rotors. ${ }^{32}$

As mentioned before, previous works tried to present an approach for predicting the unbalance and its localization, although most of them just could recognize the plane of the unbalance. Thus, this field of research is still an open area because the industries need robust and accurate techniques for this purpose. The current study attempts to establish a method which can localize the unbalanced mass between various discs (planes) and approximate eccentric mass and radius values via the artificial neural network. The ANN has been selected as a predictor tool due to its learning property and its application in the area of vibration. ${ }^{33,34}$

\section{METHODOLOGY}

In the balancing operation of rotary systems, identifying the plane on which an unbalanced mass is located, radius and mass values are necessary. To attain this purpose, an ANN model has been established to verify this idea. Whereas ANN requires examples set for training, a rotary system with multi-discs was simulated to generate the proper data.

\subsection{Simulation and Data Analysis}

Firstly, a shaft with four discs was modelled as a rotor by ADAMS Software. As illustrated in Fig. 1, the rotor is placed on the base plate by two simple ball bearings, and the base plate was constrained by 12 springs. So, the plate has six degrees of freedom (6 DOF). The rotor, discs, and base plate are rigid and solid, though the springs show flexible behaviour. The diameter of the shaft is $0.02 \mathrm{~m}$, and the discs' radius and thickness are $0.13 \mathrm{~m}$ and $0.02 \mathrm{~m}$, respectively. The system was rotated by $20 \mathrm{~Hz}$ angular speed value, and various eccentric masses with different distances to the rotor centre were attached on different disc numbers. In each case, the accelerations of both bearings were recorded by the software. The eccentric radiuses were 6,8 , and $11 \mathrm{~cm}$, and unbalanced masses were $10,15,20$, and 25 gr. Therefore, vibration data were obtained for the 48 samples. Out of that data, $60 \%$ has been used as a training example, $20 \%$ and $20 \%$ were considered as test and evaluation steps, respectively. Finally, the time

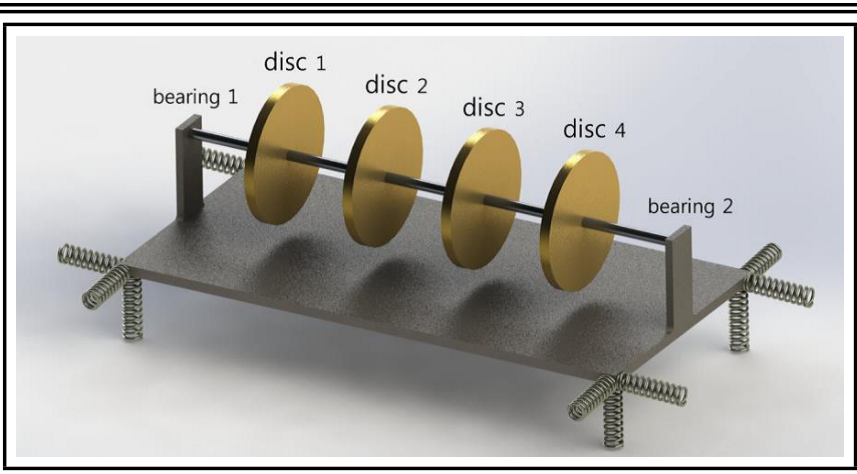

Figure 1. The simulated rotary system.

domain accelerations were converted to frequency domain by Fast Fourier Transformation (FFT).

For example, a 20 gr. unbalance mass with an $11 \mathrm{~cm} \mathrm{ec-}$ centric radius was situated on the disc number 1 , next number 2 , then 3 , and lastly on disc number 4 . The amplitude of the vertical accelerations in frequency domains were plotted at the bearing number 1 for all four discs and are shown in Fig. 2.

Based on the results represented in Fig. 2, whenever the unbalanced mass has a longer distance from the first bearing, the peak of the produced vibration has a lower value. In fact, this concept can be applied by the ANN to identify the disc number that is generating vibration due to the unbalance.

\subsection{Architecture of ANN}

Artificial neural networks stimulated from human brain are generally presented as unit of intersected "neurons" which can compute values from inputs, and are able of machine learning due to their adaptive nature. A single neuron processes multiple inputs and generates an output resulting in the utilizing of an activation function to a linear combination of the inputs:

$$
y_{i}=\varphi_{i}\left(\sum_{j=1}^{N} \omega_{i j} x_{j}+b_{i}\right)
$$

where $\left\{x_{j}\right\}$ is the set of inputs, $\omega_{i j}$ is the synaptic weight connecting the $j$ th input to the $i$ th neuron, $b_{i}$ is a bias, $\varphi_{i}()$ is the activation function, and $y_{i}$ is the output of the $i$ th neuron considered. In Fig. 3, the schematic topology of ANN is exemplified.

In this application of the ANN, the aim is that acceleration of the bearing is considered as an input signal. The unbalance mass, disc number (plane) which has an unbalanced mass and eccentric radius is the outputs of the ANN. The feed-forward ANN structure was employed, and it was constructed in MATLAB Software. Various topologies of the ANN include of different hidden layers and different numbers of neurons that were assessed in terms of performance. The input signal consists of the first bearing's acceleration in three directions. Fig. 4 illustrates the proposed ANN model for the determination of the unbalance parameters.

The architecture and achieved accuracy of the ANN model will be stated in next part. Three parameters are checked: finding the disc number which has eccentric mass, prediction of 


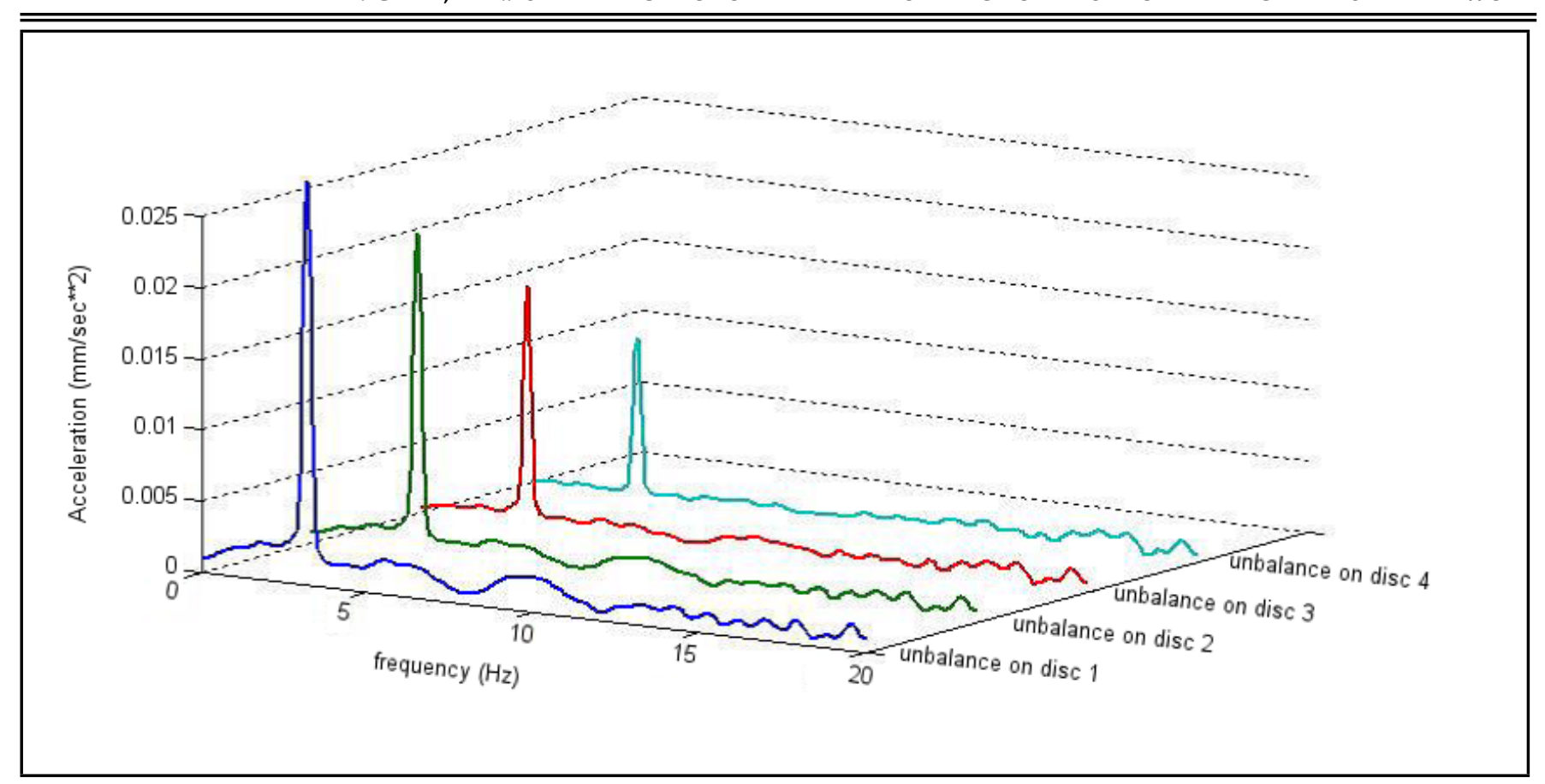

Figure 2. Frequency responses of first bearing through different location of unbalanced mass (20 gr).

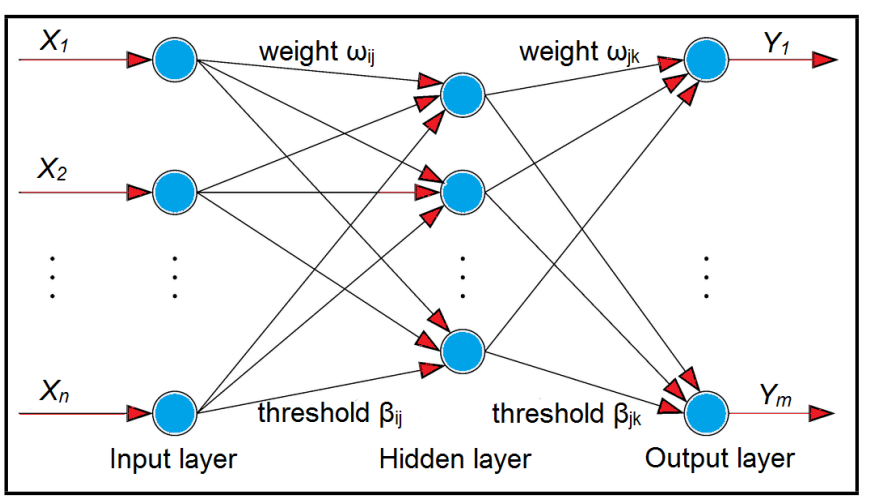

Figure 3. Schematic architecture of ANN.

eccentric mass, and the radius values.

\section{RESULTS AND DISCUSSION}

Various ANN topologies were assessed in performance by testing different numbers of neurons and different hidden layers. Also, other types of ANN such as perceptron, Hopfield, Recurrent etc. were tested, but feed-forward back-propagation showed better performance. The input and output sets were exerted into the network. After training and adapting of the ANN was done by a Levenberg-Marquardt (LM) algorithm, the best architecture has been obtained by 10 hidden layers with 10 neurons in each layer. From that, $60 \%$ of the data was used in the training step, and $20 \%$ and $20 \%$ of the data have been utilized in the test and evaluation process, respectively. The ANN was reached after 50 epochs. The weights of the neurons were reached during training of the ANN algorithm in the software. In fact, a closed loop was run to minimize the difference between the target and the ANN output. Weights are adjusted to reach minimum difference.

Subsequently, the accuracy of the ANN in the prediction
Table 1. The accuracy of the ANN model in predictions.

\begin{tabular}{|c|c|c|}
\hline $\begin{array}{l}\text { Prediction of the disc number } \\
\text { which has unbalanced mass }\end{array}$ & $\begin{array}{l}\text { Prediction of the } \\
\text { eccentric radius }\end{array}$ & $\begin{array}{l}\text { Prediction of the } \\
\text { eccentric mass }\end{array}$ \\
\hline $96 \%$ & $96 \%$ & $94 \%$ \\
\hline
\end{tabular}

purpose was verified. For this goal, all of the 48 examples were entered as the inputs, and the outputs of the ANN were compared to the actual values. The Fig. 5 (a), (b), and (c) exemplifies this comparison between the actual value to that of the predicted value by the ANN model for the disc number, the eccentric radius, and the mass values, respectively. Table 1 represents the accuracy of the ANN in predictions. As can be seen in Fig. 5 and Table 1, the best accuracy of prediction has been reached in eccentric radius, and the lowest was acquired for the unbalance localization.

As can be seen, the ANN model accuracy in prediction of the eccentric mass is low, but in the disc number and the eccentric radius are around $96 \%$ accurate. Thus, by this ANN architecture, the accuracy of all outputs including the mass value, the disc number, and the eccentric radius are higher than 90\%. The correctness of the proposed ANN model compared to the earliest presented model in Walker et al. is a little lower, though the current ANN model has the ability to predict the radius and the mass value of the unbalance with acceptable accuracy. ${ }^{30}$ As stated in a literature survey, in active rotor balancing, identifying the location of the unbalanced mass with good accuracy and online output is very useful. ${ }^{31,32}$ This achieved ANN model can be applied in this aim. Most of the former established ANN models generally were created for fault classification, ${ }^{24-29}$ but some of them were employed to identify unbalance parameters. ${ }^{30,33}$ 


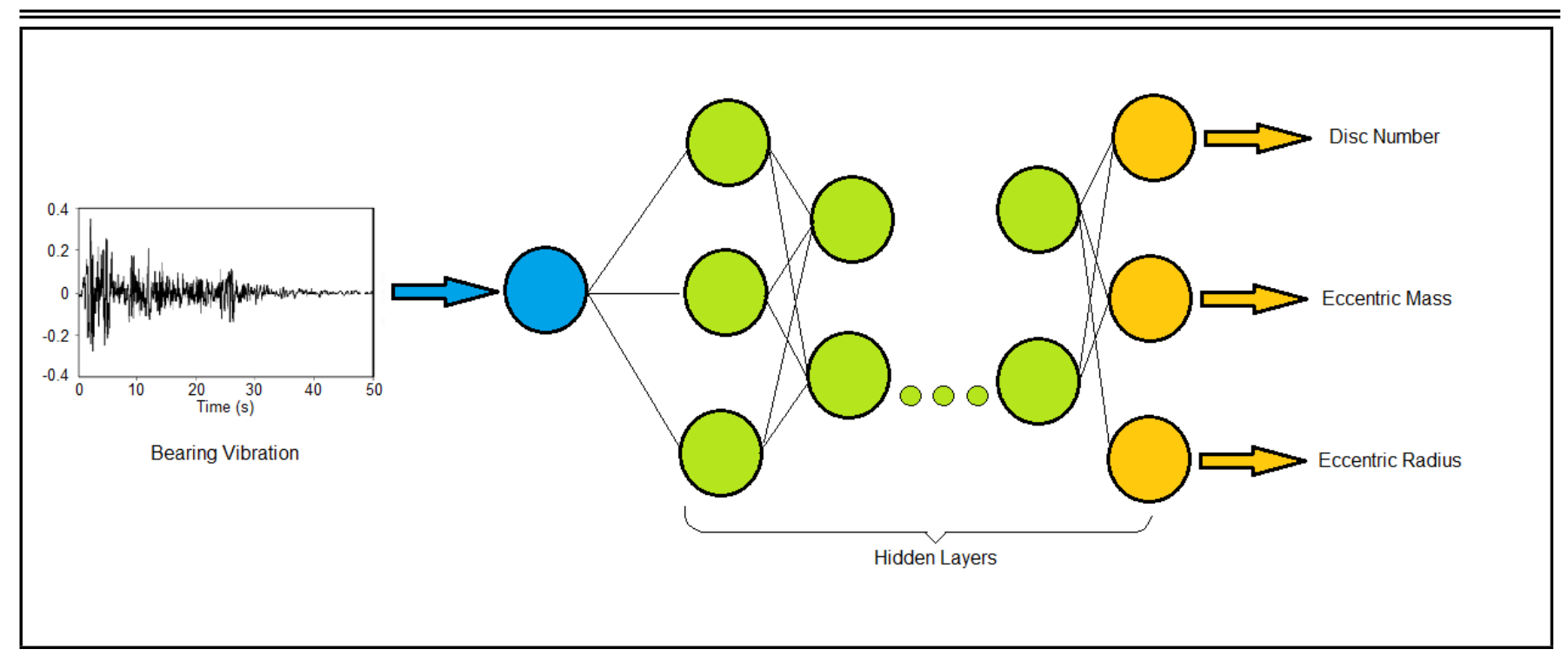

Figure 4. Proposed ANN model for unbalance parameters identification.

\section{CONCLUSIONS}

As mentioned before, in rotary systems, elimination of harmful vibrations produced by an unbalanced mass is essential, and to remove these vibrations requires some parameters about unbalance, such as the unbalance mass value and the location of it. In this study, a novel technique was utilized to detect and predict the unbalance rotor parameters. To acquire this objective, an ANN model has been unveiled which can predict the location and value of the eccentric mass. The reason the ANN was selected as the intelligent tool is having the ability to model by nonlinear characteristics. The trained ANN by a LM algorithm with 10 hidden layers and 10 neurons in each layer presented the best performance in this application. The potential accuracy of the ANN in the prediction of finding the disc which has an unbalanced mass and the location of it was between 94 to $96 \%$. It seems that the accuracy of the proposed model demonstrates good accuracy, and can be developed for industrial purposes. In future study, a test rig will be constructed to apply this concept.

\section{REFERENCES}

1 Bently, D.E. Fundamentals of Rotating Machinery Diagnostics, ASME Press, Canada, (2002), 1st ed. https://dx.doi.org/10.1115/1.801FRM_ch5

2 Jennions, I. K. Integrated vehicle health management: perspectives on an emerging field, SAE International, Warrendale, Pennsylvania, (2011). https://dx.doi.org/10.1002/jsc. 1976

3 Hassan, M. A., Coats, D., Gouda, K., Shin, Y. J., and Bayoumi, A. Analysis of nonlinear vibration interaction using higher order spectra to diagnose aerospace system faults, Proc. Aerospace Conference, Big Sky, Montana, (2012). https://dx.doi.org/10.1109/AERO.2012.6187370

4 Ganeriwala, S. N., Schwarz, B., and Richardson, M. H. Op- erating deflection shapes detect unbalance in rotating equipment, Sound and Vibration, 43 (5), 11-13, (2009).

5 Xue, H., Wang, H., Song, L. and Chen, P. Structural fault diagnosis of rotating machinery based on distinctive frequency components and support vector machines, Proc. International Conference on Intelligent Computing, Zhengzhou, China, 341-248, (2011). https://dx.doi.org/10.1007/978-3-642-25944-9_44

6 Han, T., Bai, J., Yin, Z. J. Dynamic balancing simulation based on virtual prototyping technology, 8th International Conf. on Reliability, Maintainability and Safety, Chengdu, China, 1035-1039, (2009). https://dx.doi.org/10.1109/ICRMS.2009.5269991

7 Jiao, W., Yuan, Q., Chang, Y. Study on the coupled bending-torsional vibration of unbalanced rotor system with external excitations, 2012 IEEE International Conference on Computer Science and Automation Engineering (CSAE), Zhangjiajie, China, (2012). https://dx.doi.org/10.1109/CSAE.2012.6272609

8 Abdul-Aziz, A., Woike, M. R., Oza, O., Mathhews, B., Lekki, J. Rotor health monitoring combining spin tests and data-driven anomaly detection methods, Journal Structure Health Monitoring, 11 (1), 3-12, (2012). https://dx.doi.org/10.1177/1475921710395811

9 Abdul-Aziz, A., Woike, M. R., Baaklini, G. Y. Turbine engine disk health monitoring assessment using spin test data, Smart structures and materials \& nondestructive evaluation and health monitoring, San Diego, California, (2012). https://dx.doi.org/10.1117/12.912524

10 Edwards, S., Lees, A., Friswell, M. Fault diagnosis of rotating machinery, Shock and Vibration Digest, 30 (1), 4-13, (1998). 


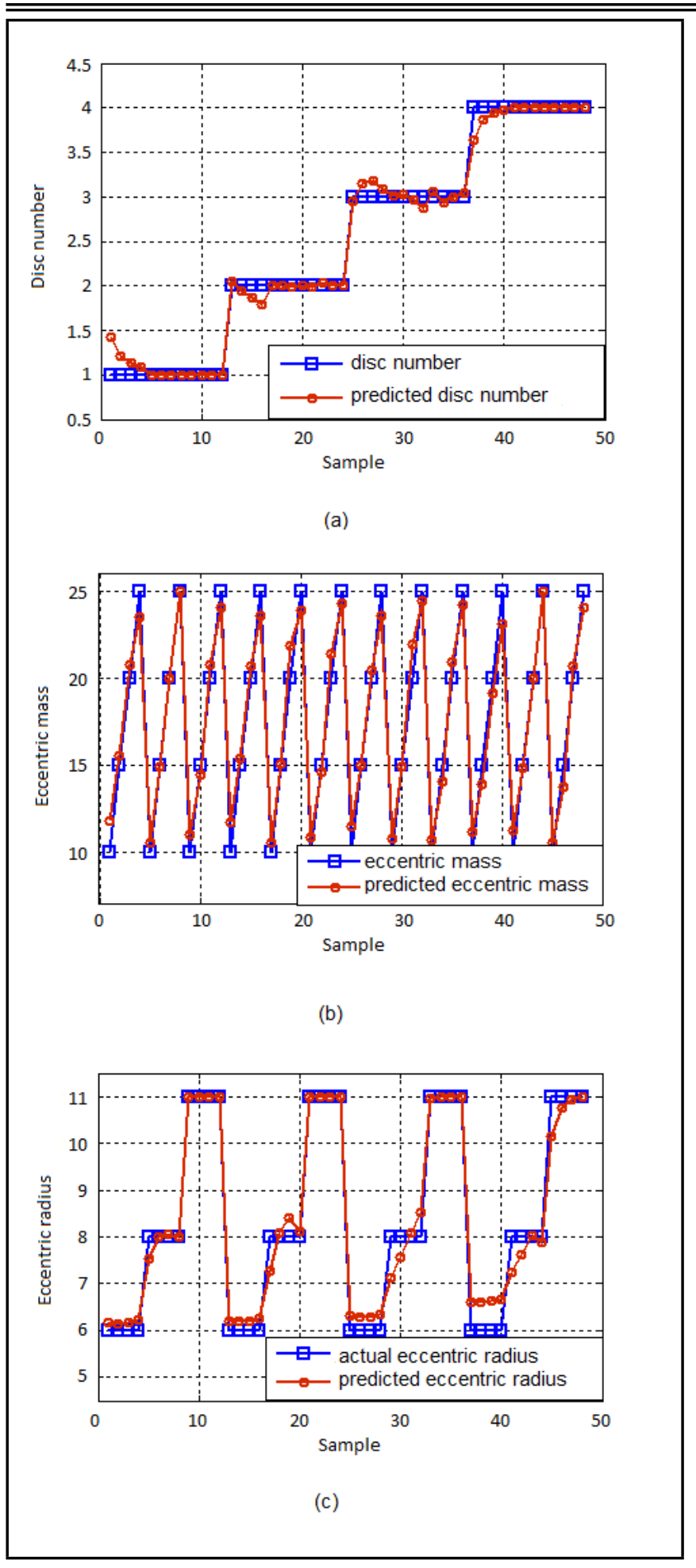

Figure 5. The comparison between actual and predicted by ANN model values: (a) prediction of the disc number which has unbalance mass, (b) the eccentric radius value, (c) the eccentric mass value.

11 Foiles, W. C., Allaire, P. E., Gunter E. J. Review: rotor balancing. Shock and Vibration, 5 (5-6), 325-336, (1998). https://dx.doi.org/10.1155/1998/648518

12 Randall, R. B., State of the art in monitoring rotating machinery_part 1, Sound and Vibration, 38 (3), 14-2, (2004).

13 Mosaad, M. I., and Salem, F. LFC based adaptive PID controller using ANN and ANFIS techniques, Journal of Elec- trical Systems and Information Technology, 1 (3), 212-222, (2014). https://dx.doi.org/10.1016/j.jesit.2014.12.004

14 Krodkiewski, J. M., Ding, J., Zhang, N. Identification of unbalance change using a non-linear mathematical model for multi-bearing rotor systems, Sound and Vibration, 169 (5) 686-698, (1994). https://dx.doi.org/10.1006/jsvi.1994.1041

15 Lees, A. W., Friswell, M. I. The evaluation of rotor imbalance in flexibly mounted machines, Sound and Vibration, 208 (5), 671-683, (1997). https://dx.doi.org/10.1006/jsvi.1997.1260

16 Sinha, J. K., Friswell, M. I., Lees, A. W. The identification of the unbalance and the foundation model of a flexible rotating machine from a single run-down, Journal Mechanical Systems and Signal Processing, 16 (2), 255-271, (2002). https://dx.doi.org/10.1006/mssp.2001.1387

17 Saleem, M. A., Diwakar, G., Satyanarayana, M. R. S. Detection of unbalance in rotating machines using shaft deflection measurement during its operation, ISOR Journal of Civil and Mechanical Engineering, 3 (3), 8-20, (2012). https://dx.doi.org/10.9790/1684-0330820

18 Walker, R. B., Perinpanayagam, S., Jennions, I. K. Physics-based simulation for health management of rotating machinery, 8th International Conference on Condition Monitoring and Machine Failure Prevention Technologies, Cardiff, Wales, (2011).

19 Walker, R. B., Perinpanayagam, S., Jennions, I. K. Simulating unbalance for future IVHM applications, The 12th International Modal Analysis Conference, Jacksonville, Florida, (2012). https://dx.doi.org/10.1007/978-14614-2431-4_15

20 Mottershead, J. E., Friswell, M. I., Mares, C. A method for determining model-structure errors and for locating damage in vibrating systems, Mecchanica, 34 (3), 153-166, (1999). https://dx.doi.org/10.1023/A:1004684417925

21 Feldman, M., and Seibold, S. Damage diagnosis of rotors: application of Hilbert transform and multihypothesis testing, Vibration and Control, 5 (3), 421-442, (1999). https://dx.doi.org/10.1177/107754639900500305

${ }^{22} \mathrm{Li}$, B., and Chow, M. Y. Detection of common motor bearing faults using frequency-domain vibration signals and a neural network based approach, American Control Conference, Philadelphia, Pennsylvania, (1998). https://dx.doi.org/10.1109/ACC.1998.702983

23 Li, B., Chow, M. Y., Tipsuwan, Y., Hung, J. C. Neuralnetwork-based motor rolling bearing fault diagnosis, IEEE Transaction on Industrial Electronics, 47 (5), 1060-1069, (2000). https://dx.doi.org/10.1109/41.873214 
${ }^{24}$ Tao, Y., and Qingkai, H. Crack fault identification in rotor shaft with artificial neural network, Sixth International Conference on Natural Computation, Yantai, China, (2010). https://dx.doi.org/10.1109/ICNC.2010.5583771

${ }^{25}$ Qiu, Y., and Rao, S. S. A fuzzy approach for the analysis of unbalanced nonlinear rotor systems, Sound and Vibration, 284 (1), 299-323, (2005). https://dx.doi.org/10.1016/j.jsv.2004.06.029

${ }^{26}$ Barakat, M., Druaux, F., Lefebvre, D., Khalil, M., Mustapha, O. Monitoring of rotating machine by means of self-adaptive growing neural network, 19th Mediterranean Conference on Control \& Automation, Corfu, Greece, (2011). https://dx.doi.org/10.1109/MED.2011.5983017

27 McCormich, A. C., Nandi, A. K. Real-time classification of rotating shaft loading conditions using artificial neural networks, IEEE Transaction on Neural Networks, 8 (3), 748757, (1997). https://dx.doi.org/10.1109/72.572110

${ }^{28} \mathrm{Li}$, Y., Wang, Z., Wang, X. Fault classification of turbine-generator set based on artificial neural network, 20th International Conference on Computer Application and System Modeling, Taiyuan, China, (2010). https://dx.doi.org/10.1109/ICCASM.2010.5622561

29 Santos, F. L., Duarte, M. L. M., Faria, M. T. C. D., Eduardo, A. C. Balancing of a rigid rotor using artificial neural network to predict the correction masses, Acta Scientiarum Technology, 31 (2), 151-157, (2009). https://dx.doi.org/10.4025/actascitechnol.v31i2.3912

${ }^{30}$ Walker, R. B., Vayanat, R., Perinpanayagam, S., Jennions, I. K. Unbalance localization through machine nonlinearities using an artificial neural network approach, Mechanism and Machine Theory, 75, 54-66. (2014). https://dx.doi.org/10.1016/j.mechmachtheory.2014.01.006

31 Beltran-Carbajal, F., Silva-Navarro, G., Arias-Montiel, M. Active unbalance control of rotor systems using on-line algebraic identification methods, Asian Journal of Control, 15 (6), 1627-1637, (2013). https://dx.doi.org/10.1002/asjc.744

${ }^{32}$ Cupial, P., and Koziol, M. The analysis of a smart jeffcott rotor with direct velocity feedback control in the supercritical range, Journal of Low Frequency Noise, Vibration and Active Control, 32 (3), 205-216, (2013). https://dx.doi.org/10.1260/0263-0923.32.3.205
33 Gohari, M., Rahman, R. A., \& Tahmasebi, M. Prediction head acceleration from hand and seat vibration via artificial neural network model, Applied Mechanics and Materials, 471, 161-166, (2014). https://dx.doi.org/10.4028/www.scientific.net/AMM.471.161

${ }^{34}$ Gohari, M., Rahman, R., Tahmasebi, M., Nejat, P. Offroad vehicle seat suspension optimisation, part I: derivation of an artificial neural network model to predict seated human spine acceleration in vertical vibration, Journal of Low Frequency Noise, Vibration and Active Control, 33 (4), 429-442, (2014). https://dx.doi.org/10.1260/02630923.33.4.429

${ }^{35}$ Edwards, S., Lees, A. W., Friswell, M. I. Fault diagnosis of rotating machinery, Shock and Vibration Digest, 30 (1), 4-13, (1998).

36 Jalan, A. K., and Mohanty, A. R. Model based fault diagnosis of a rotor-bearing system for misalignment and unbalance under steady-state condition, Sound and Vibration, 327 (3-5), 604-622, (2009). https://dx.doi.org/10.1016/j.jsv.2009.07.014

37 Jamadar, I. M., and Vakharia, D. P. A novel approach integrating dimensional analysis and neural networks for the detection of localized faults in roller bearings, Journal of Measurement, 94, 77-185, (2016). https://dx.doi.org/10.1016/j.measurement.2016.07.086

38 Chih-Hao, C., Rong-Juin, S., Chih-Kao, M. Rotating machinery diagnosis using wavelet packets-fractal technology and neural network, Journal of Mechanical Science and Technology, 21 (7), 1058-1065, (2007). https://dx.doi.org/10.1007/BF03027655

${ }^{39}$ Huaqing, W., and Peng, C. Intelligent diagnosis method for rolling element bearing faults using possibility theory and neural network, Journal of Computer and Industrial Engineering, 60 (4), 511-518, (2011). https://dx.doi.org/10.1016/j.cie.2010.12.004

${ }^{40}$ Panda, S. S., and Mahapatra, S. S. Online multi-response assessment using Taguchi and artificial neural network, Journal of Manufacturing Research, 5 (3), 305-326, (2010). https://dx.doi.org/10.1504/IJMR.2010.033469 